\title{
Managing Security Requirements Patterns Using Feature Diagram Hierarchies
}

\author{
Rocky Slavin ${ }^{1}$, Jean-Michel Lehker ${ }^{1}$, Jianwei Niu ${ }^{1}$, Travis D. Breaux ${ }^{2}$ \\ Department of Computer Science ${ }^{1}$ \\ University of Texas at San Antonio \\ San Antonio, Texas, USA \\ \{rslavin, jlehker, niu\}@cs.utsa.edu \\ Institute for Software Research ${ }^{2}$ \\ Carnegie Mellon University \\ Pittsburgh, Pennsylvania, USA \\ breaux@cs.cmu.edu
}

\begin{abstract}
Security requirements patterns represent reusable security practices that software engineers can apply to improve security in their system. Reusing best practices that others have employed could have a number of benefits, such as decreasing the time spent in the requirements elicitation process or improving the quality of the product by reducing product failure risk. Pattern selection can be difficult due to the diversity of applicable patterns from which an analyst has to choose. The challenge is that identifying the most appropriate pattern for a situation can be cumbersome and time-consuming. We propose a new method that combines an inquiry-cycle based approach with the feature diagram notation to review only relevant patterns and quickly select the most appropriate patterns for the situation. Similar to patterns themselves, our approach captures expert knowledge to relate patterns based on decisions made by the pattern user. The resulting pattern hierarchies allow users to be guided through these decisions by questions, which elicit and refine requirements as well as introduce related patterns. Furthermore, our approach is unique in the way that pattern forces are interpreted as quality attributes to balance trade-offs in the resulting requirements. We evaluate our approach using access control patterns in a pattern user study.
\end{abstract}

Keywords-security; requirements; patterns; feature diagram;

\section{INTRODUCTION}

A requirements pattern is a structure that engineers can use to generate one or more requirements for a recurring situation [1]. Based on design patterns [2], each requirements pattern describes a recurring problem as well as a core solution which can be used repeatedly, but not necessarily in the same way every time. Because situations vary, a requirements pattern must be parameterized to selectively control for those effects that vary across problem spaces. For example, an engineer may choose between single sign-on [3], where users need only one username and password for logging into different systems, as means to maximize usability, where they prefer more complex role-based access control (RBAC) [4] requirements to maximize confidentiality by compartmentalizing access to information. In this example, the engineer perceives constraints differently and desires different levels of control: more control over different systems to manage logins, or more control over classification of resources into roles.

Requirements patterns incorporate engineering knowledge into the solution space of a pattern in order to provide a basis for requirements elicitation and generation. For example, patterns can itemize pre-conditions to indicate when a pattern applies to a given scenario, or questions whose answers direct the engineer from one solution to another (e.g., from single sign-on to RBAC). Such knowledge reuse allows software engineers to solve problems in a more effective manner. That is, patterns consist of tried-and-tested solutions that have been shown to be effective when applied in the correct context. Consequently, patterns serve as a common language for software engineers to document their design decisions [5].

Security requirements patterns are a special case of requirements that address security risks in a system. Historically, security is dealt with using a penetrate-and-patch approach [6], wherein security problems are identified and addressed in response to penetration testing of the fullyfunctional system, sometimes in a post-deployment situation. If the system failures are intrinsic to the design, then significant rework is required [7]. Alternatively, it is more cost effective to identify security flaws early in the requirements and design stages of development, which is the focus and intent of security requirements patterns [8].

In the wild, security requirements patterns appear mostly in isolation, either in small sets of related patterns or repositories and related only by a common topic or theme [9, 10]. Combined with the lack of guidance for pattern selection [11], engineers lack the structure needed to holistically address security and balance complex forces or quality attributes (e.g., usability and confidentiality). As the number of security patterns continues to grow [12], engineers face an increased challenge in recognizing what patterns to select [13]. The contribution of our approach is as follows: (1) we propose a new security requirements pattern format that aims to organize requirements knowledge into a canonical form that itemizes this knowledge into inter-dependent questions that investigate the problem space; (2) we demonstrate that these patterns can be linked into a hierarchy to make problem space trade-offs more salient and to connect related patterns to comprise holistic solution spaces; and (3) we evaluate this new format and hierarchy in a user study to measure speed and correctness in selecting patterns to apply to example scenarios.

We evaluate our approach in a user study consisting of graduate and undergraduate students at the University of Texas at San Antonio (UTSA). The study examined our method's ability to deduce the most appropriate set of patterns by having novice users with limited security knowledge and experience use a pattern hierarchy to select patterns for a scenario. We then compared the results with selections that experts chose for the same scenario. Based on our analysis, we found that novice users not only were more accurate in their pattern selections, but were able to select patterns more quickly than users 
without a hierarchy. From these results and further observations we assert that the use of pattern hierarchies can improve the usability of security requirements by providing a means for easier access and faster selection as well as better documentation of related patterns.

The remainder of this paper is organized as follows: in Section II, we review the theoretical foundations and background upon which we based our approach; in Section III, introduce our requirements pattern template and in Section IV, we present our pattern hierarchy; in Section V, we present our empirical study design with our results and observations presented in Section VI; finally, we present related work in Section VII, with future work in Section VIII and our conclusion in Section IX.

\section{THEORETICAL FOUNDATIONS}

Our approach is based on prior work in pattern languages, feature diagrams and the requirements elicitation, which we now discuss.

\section{A. Pattern Languages}

Alexander et al. introduced the earliest notion of pattern as a structured device for reusing knowledge in seminal work on building architecture patterns [2]. More recently, there has been substantial work on object-oriented design patterns [14], requirements patterns $[9,15]$ and security patterns $[10,12$, 16]. A security requirements pattern provides a software engineer with a reusable set of requirements to solve common security problems. To be effective, a requirements pattern should incorporate an engineer's knowledge about their system context to select the most appropriate requirement. For example, many systems use passwords to restrict access to sensitive resources (e.g., healthcare data, bank account data, and purchase histories) as well as to associate unique identifiers with individual users (e.g., forums or social networking sites). The risk to individual users of having their password retrieved through a security attack is different depending on the harms of a data breach. A breach to a bank account could be more expensive than the cost of a breach to web forum, where the attacker could at best post derogatory comments. Therefore, an engineer needs a means to tailor reusable knowledge to their situation to yield requirements that balance complex and sometimes competing forces (e.g., performance, security, usability, etc.). Despite this need, current security patterns are not configurable or linkable to enable engineers to tailor security requirements to their needs.

\section{B. Feature Diagrams}

A feature diagram is a graphical notation to describe how products can be composed from multiple features [17]. A feature is an increment of functionality, usually with a coherent purpose [18]. Features may be linked together in a feature diagram to describe common and variable requirements in a system composition [19]. The links between features may be mandatory or optional, and groups of features can be organized using logical connectives such as inclusive or exclusive or. Figure 1 shows an example including the most common notations used in a feature diagram as well as their meanings.

Figure 1 describes an example. If we were to describe access control requirements, a mandatory feature would be authentication [12]. An optional feature could be the inclusion of a role-based access control policy. We can further break down some features into groups of features. Here, we can make a decision between the optional mandatory access control (MAC) [20] and discretionary access control (DAC) [21]. We could choose one or the other, but not both, denoted by Exclusive Or. Similarly, we must choose between using passwords or biometrics for authentication. In this case, Inclusive Or, it is possible to have both, but at least one must be included.

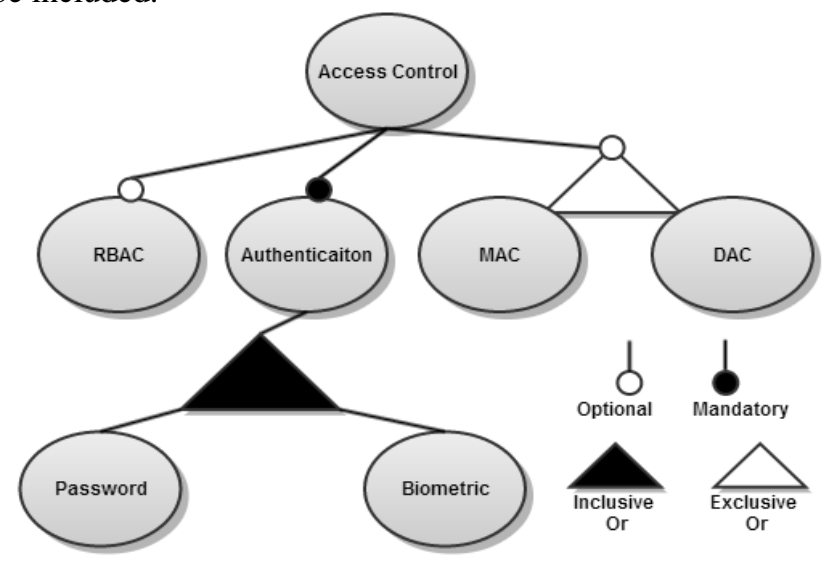

Figure 1. Feature diagram notation

We felt that feature diagrams would be a suitable notation for visually representing pattern hierarchies because security concepts have mandatory and optional features. By breaking down the features of security concepts, we can construct a diagram using logical connectives which can help a software engineer understand which features are most important for their specific application. Once features associated with the security concept are identified, requirements generation can be customized to the user's specific situation. Recently, the notation has been given a formal semantics to enable reasoning about complex combinations of features and potential conflicts [22]. This may be something we can leverage in the future.

\section{Inquiry-Cycle}

The Inquiry Cycle Model (ICM) by Potts et al. [23] is a requirements elicitation method for refining requirements by generating discussion through questions. This model is used to analyze requirements of a system through questions and answers which describe the solutions. We adapt this idea of using ICM questions by using the answers to "how", "why", "what", and "when" questions to direct users to the requirements pattern that will provide them with the resources needed to generate complete requirements. If a user was applying access control, they would start with the Access Control pattern and answer a question about how access to a resource is decided. If the user answers something like "the resource's owner decides who should have access to it," they would find the DAC pattern most suitable. Their answer to this question helped them select the correct type of pattern for their needs. This is a very simple example and not all situations will be so clearly outlined.

The ICM assists in the creation of pattern hierarchies to help users map their needs to relevant patterns in the context of their analysis. Section V describes how we were able to build and refine our hierarchy by interviewing security experts with 
the ICM. We encouraged experts to ask questions when dealing with hypothetical situations involving security. The experts continued to refine the requirements for the scenarios by asking questions for clarification. By "forcing" them to use an approach similar to the ICM, we were able to elicit the kinds of questions that would be used to connect patterns within hierarchies.

\section{PATTERN TEMPLATE}

The presentation of pattern content varies depending on the pattern author. In requirements engineering, formal and semiformal methods, such as Tropos [24] and Problem Frames [25], structure requirements knowledge into pattern-like representations. However, many approaches that principally identify themselves as patterns use natural language templates (NLT) to illustrate patterns [26, 27]. These NLT approaches tend to base their template on the so-called Gang-of-Four (GoF) [14] book which outlines the following essential pattern elements:

- Pattern Name - A simple phrase to describe the problem

- Problem - A description of when to apply the pattern

- Solution - A general arrangement of the elements used to solve the problem

- Consequence - The results and trade-offs of applying the pattern

Our approach includes two additional important features: forces, which determine quality attributes that are impacted (maximized, minimized, etc.) by the pattern, and relations or links to other patterns using an inquiry-based approach. For example, the access control requirements pattern may contain such forces as generalizability, flexibility, and modifiability since they would be particularly relevant to its application and a question such as, "How are authorization privileges bound to actors and resources?” may lead to an authentication pattern.

By balancing forces using questions which invoke discussion and refinement of security requirements, we aim to minimize the negative consequences or liabilities of the pattern (e.g., access control may require many rules and increase the system's complexity) as well as capture the applicability of the pattern [28]. The use of an approach based on the ICM provides a way to connect existing security requirements patterns as well as refine system requirements themselves through questions that surface hidden assumptions about the system and its environment. The approach works on two levels: (Level 1) questions relevant to the problem can either draw out specific information from the situation which can be applied to generic requirements in the template in order to generate specific requirements, or (Level 2) they relate the pattern to other patterns relevant to the situation as a means to increase requirements coverage by identifying dependencies or to introduce alternative requirements that balance forces in alternative ways that may be better suited to a particular problem or stakeholder needs. Pattern hierarchies are constructed using the second level, which further supports eliminating unnecessary or unfitting requirements to select the most appropriate pattern.

To catalog patterns, we used a standardized pattern template consisting of six elements. The template we used is an elaboration of ideas devised at RePa'12 and our previous work on the Standardized Pattern Format for deriving characterizations and boundaries of patterns [29]. Using this pattern template is what enables us to construct a pattern hierarchy. To illustrate the different aspects of the template, we use access control as an example.

The template consists of the following elements:

- Name - a unique name that is limited to the scope of the pattern

- Problem - a statement of the problem to be addressed or a high-level goal to be achieved

- Context - domain assumptions that must be true in conjunction with the generated requirements

- Forces - the non-functional quality attributes that create trade-offs for the application of the pattern

- Solution - a set of questions which refine requirements and guide the pattern user to related patterns

- Management - additional information related to the pattern including examples and known uses

We now describe the use of each of the attributes using access control.

\section{A. Name}

For our running example, we name the pattern "Access Control" to describe what the pattern covers. In addition, it can be uniquely incorporated into the pattern hierarchy.

\section{B. Problem}

The problem shall be expressed either as the security objectives that need to be achieved or the threats that must be mitigated. For our example, we define the problem as "the confidentiality and integrity of resources shall be protected by regulating access to the resources based on different factors.” Here, confidentiality and integrity, [30], two fundamental security attributes, are addressed.

\section{Context}

A pattern addresses a generic problem in a specific context [12], which shall describe the nature of the situation. This includes any domain assumptions as well as expectations of the system and its environment. For access control we describe the context as "any computer-driven environment in which the access to the resources needs to be regulated” and we list the following assumptions:

- The administrator involved in implementing the authorization system shall be trusted.

- The actors involved in granting or denying authorization shall have the ability to restrict access to the resources being protected.

- Actors shall not assume the identity of other actors with different rights.

We also include one expectation:

- Actors will not circumvent the system to gain access to resources.

\section{Forces}

Forces that must be balanced to make a decision are necessary for the creation of requirements in order to provide boundaries and limits. We believe it is useful to list these potentially conflicting or complementary goals as quality attributes in order to better balance the trade-offs of benefits 
and cost that are imposed by the requirements [31]. For access control we list three forces:

- Generalizability - The authorization structure must be independent of the type of resources.

- Flexibility - The authorization structure shall be flexible enough to accommodate different types of principals (users or subsystems), objects, and rights.

- Modifiability - It should be easy to modify the rights of a principal in response to changes in his or her or its duties or responsibilities.

\section{E. Solution}

To solve the problem and balance the forces, the solution includes a series of ICM-inspired questions that help elicit responses from a user or a subject matter expert. A set of requirements templates with variable parts may be configured by the developer and may correspond directly to the questions. The answers to the questions may be pre-conditions to asking additional questions, pieces to fill in the requirements templates, or guides to other patterns. The questions for our example are:

- Which entities (principals) exist in the system and what resources do they need to access?

- Can two entities access the same resource at the same time?

- How are the resources intended to be accessible?

- Can users of the system be categorized into roles that will have different access privileges? See Role-Based Access Control pattern.

- Can resources be assigned labels by the system so that users are given clearance to access resources based on levels of clearance? See Mandatory Access Control pattern.

- Can access to resources be regulated by the owner of the resource? See Discretionary Access Control pattern.

- How are entities to be authenticated in order to gain access to the system? See Authentication pattern.

The requirements template for our example consists of:

- $\quad<$ list of entities > shall < be permitted | not be permitted> to access $<$ resource $>$ simultaneously.

- $\quad<$ list of entities $>$ shall access < resource $>$ through the use of $<$ action $>$.

- An entity shall be granted or denied privileges to $<$ resource> based on < authorization criteria $>$

Some questions are accompanied by italicized text. This text refers a pattern-user to a corresponding pattern $[9,12]$ for the question (as seen in Figure 1). The requirements template also lists generic requirements which can be refined based on the answers to some of the questions. For these requirements, words surrounded by " $<$ " and ">” are mandatory parts of the requirements which must be filled in and words surrounded by "[" and "]" are optional. In some cases, a choice of possible answers are provided separated by the "|" character.

\section{F. Management}

Finally, a pattern should include any information regarding the source of the pattern, the version, known uses, or any information relating to how the pattern was derived. For this pattern we note that it was adapted from the security design pattern from [12] and modified for requirements based on Withall's work on access control requirements [9].

\section{PATTERN HIERARCHY}

Pattern relations are visualized with feature diagrams, which connect patterns in places where a question might lead a user to another pattern. The resulting hierarchy partitions a larger problem or security area into smaller problems (i.e. patterns). We believe such a hierarchical structure would reduce the time and difficulty needed to select an appropriate pattern and security requirements for a complex situation. Furthermore, the hierarchy enables users to easily discover related patterns which could be implemented to enhance their system security.

Based on the solution space of the template, a pattern hierarchy can be derived as seen in Figure 1. The hierarchical relations between patterns appear within each pattern template as the questions that help the pattern user make decisions on which pattern to include for their situation. For the Access Control pattern in Section III, the question, "how are entities to be authenticated in order to gain access to the system?” is associated with the Authentication pattern. Figure 1 shows this connection with a link between the Access Control and Authentication patterns. Our approach is inspired by the ICM which uses inquiry-based discussion between stakeholders in order to revise previous iterations of requirements and introduce relevant patterns. Our approach begins by highlighting existing challenges for the problem (i.e. the forces) and uses questions to elicit security requirements as responses to those challenges.

We continue to use access control as an example to illustrate a hierarchy due to its large amount of related work including various aspects and specialized models [32, 33, 34, 35]. Not only is it a commonly understood aspect of security, but it also includes many facets which allow us to demonstrate the different benefits of using a feature diagram to illustrate the pattern. In Section III we have described a single Access Control pattern from which our hierarchy extends. In this example, the access control pattern is placed as the root of the hierarchy; hence, we name the entire hierarchy after it.

In the following subsections, we describe the use of each notation for our hierarchy design using the same example from Figure 1.

\section{A. Optional}

The notation for optional components is denoted by an unfilled circle on the end of the connection closest to the optional pattern. Such components are directly related to the inquiry-based approach. In Section III-E, our example included the question, "Can users of the system be categorized into roles that will have different access privileges?" which related the Access Control pattern to the Role-Based Access Control pattern. For such an instance where the answer to the question determines whether or not the related pattern is relevant, an optional connection would be made. 


\section{B. Mandatory}

Mandatory components are denoted by a filled circle on the end of a connection closest to the mandatory pattern. In Figure 1, the Access Control pattern includes the Authentication pattern which is commonly viewed as a necessary component of access control [9] as a mandatory feature. Mandatory components do not stem from "yes" or "no" questions as such questions would imply a noncompulsory relation. Instead, such relations are made through "how" questions. For instance, we asked, "How are actors to be authenticated in order to gain access to the system?" Such a question requires a direct answer and cannot be avoided. That is, there must be a way for the actors to be authenticated. This question included a reference to the Authentication pattern and is connected within the diagram through the use of a mandatory connection.

\section{Inclusive Or}

Some questions may require a child pattern to be included, but allow for multiple children to be included simultaneously. Our example includes two forms of credential verification patterns: Password and Biometric. These patterns are included as an Inclusive Or decision since it is acceptable to use multiple credential verification mechanisms and at least one is required. These relations have a filled triangle spanning the edges between the nodes in the decision. We augment the general feature diagram notation with the possibility for brackets in the Inclusive Or relation representing a range. For instance, "[2,5]" would indicate that between 2 and 5, inclusive, of the patterns across the relation must be chosen. A single number within brackets (e.g., "[2]") would indicate exactly two must be chosen.

\section{Exlusive Or}

Similarly to the inclusive-or case, the exclusive-or relation allows for decisions between different patterns. However, this relation is used when only one choice may be made. These relations have an unfilled triangle spanning the edges between the nodes in the decision. The Access Control node illustrates the use of an Exclusive Or decision between the MAC and DAC patterns. An unfilled triangle joins their edges to signify that only one can be chosen if this optional branch is selected.

\section{EMPIRICAL STUdy DESIgN}

We believe that pattern hierarchies combined with the ICM will improve users' ability to select appropriate patterns and requirements faster and more effectively than unassisted pattern selection. Furthermore, the use of pattern hierarchies should allow novice software engineers with limited experience to benefit from expert knowledge embedded in such patterns and hierarchies.

To evaluate our approach we conducted a study to compare the pattern selection of two groups: one with a pattern hierarchy and one without a pattern hierarchy. The first step was to gather existing security patterns into a repository [36]. This assessment included design, architectural, and requirements patterns so we could better understand how different security concerns were addressed at different development stages among the pattern landscape. Sources included a literature review of textbooks and scientific publications on security patterns. A total of 143 security patterns, 30 of which we classified as security requirement patterns, were collected. We then gathered a set of patterns relating to access control from the repository to create the initial draft of a pattern hierarchy. We mapped these existing patterns to match our pattern template described in Section III in order to build the pattern hierarchy.

Next, we reached out to security experts from the local security community with the intention of revising our hierarchy and preparing for the evaluation of the hierarchy with novice users. Two banking scenarios (described in Section VI) regarding access control were presented to these experts and used as a means to gather more information and possible patterns for our existing hierarchy example. From transcriptions of expert interviews conducted using the ICM and feedback directly from the experts, we further refined the Access Control hierarchy. For each of the two scenarios we were also able to assemble a subset of patterns in the hierarchy which were most applicable. These subsets were used later in evaluation with novice users.

Finally, we evaluated the Access Control hierarchy comprised of patterns chosen by the experts by observing what patterns novices selected from it. This evaluation consisted of a comparison of novices both with and without the use of the hierarchy so as to test our hypothesis that the hierarchy would improve users' ability over unassisted pattern selection.

\section{A. Scenarios}

The two scenarios used in our study involved access control in some way due to the common use of access control as an example for security [35]. This allowed for less time to be taken in training since it would be more likely for both experts and novices alike to have some background knowledge in the domain. The scenarios involved a fictional credit union and bank which were partnering with each other. This partnership required new software to be implemented to accommodate shared data. Our description of the entities described the number of employees, their jobs, and features from each of the entities' existing software infrastructures (e.g., instant messaging system and administrative tools).

Scenario 1 described the ATM system currently in place for both institutions and gave the new requirements for the partnership. These requirements outlined the location, hours of operation, and usage fees for customers of each institution. A general set of auditing requirements were provided as well as the stipulation that only a subset of the bank's ATMs would be accessible to credit union customers.

Scenario 2 described a computerized banking system with online banking. The system would need to be implemented only for the credit union, but with possibility for relevant data to be transferred to the bank. Specifications pertaining to user access, financial transaction services, customer services, and browser support were described.

These scenario descriptions were intended to include only high-level descriptions of the institutions' requirements so as to elicit questions and discussion from the experts. This would allow us to infer more about the thought process of the experts interviewed. For situations where clarification on the scenarios was requested, a third-party trained as a domain expert representing the financial institutions was provided. The 


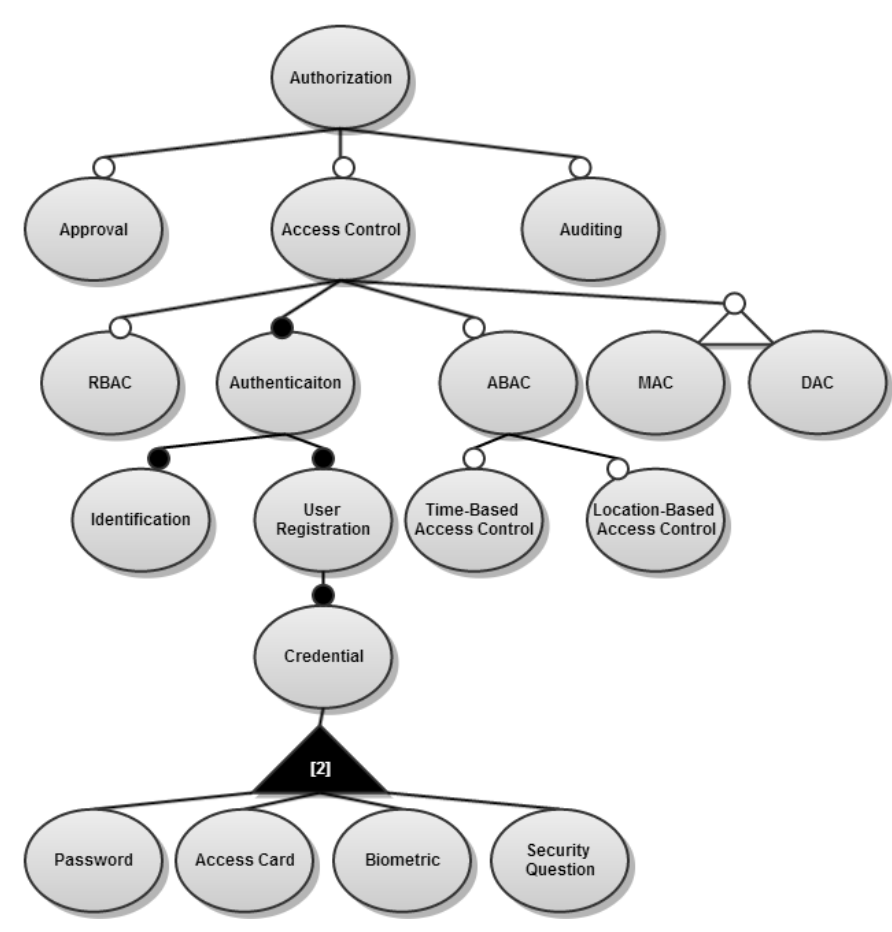

Figure 2. Access control requirements hierarchy

domain expert explained the different features required for employees based on their roles. For any other questions, we only required that the domain expert be consistent with his responses so as to provide a constant experience for each expert.

\section{B. Sampling}

We gathered expert participants with experience relating to computer security from academia and industry. We were able to recruit two members of a local security forum in San Antonio. Members of this forum were industry security specialists in the financial, utility, and local government sectors. We also enlisted participation from a member of a local application security consulting firm, a researcher and professor in the area of access control UTSA, a senior security staff member at a multinational computer technology corporation, and two post-doctoral researchers in the areas of privacy and security policies. In total, we interviewed seven industry and academic experts. Our goal was not to provide a complete representation of the security community's opinion, but instead to create an example to test the use of a hierarchy. For this reason, the representation given by our small sampling of security experts would be sufficient.

The second group consisted of university students with computer science backgrounds. We recruited participants in three undergraduate (junior and senior level) and one graduate-level computer science course. For each class, we gave a three minute explanation of what participants would be asked to do for our study and offered a $\$ 15$ gift card as compensation for time. We were able to recruit 34 participants in this way. Due to the course requirements of the department, these students also had some knowledge of computer security. We chose students as subjects due to their fresh entry-level knowledge in access control with little industry experience. This would allow us to see whether pattern hierarchies are useful in relaying expert experience in order to make better and faster decisions.

\section{Expert Interviews}

We interviewed each expert with an existing hierarchy already created from patterns gathered from textbooks and our pattern repository [9, 12, 36, 37, 38]. Our goal was to further expand and refine our hierarchy as well as to find a unique subset of the patterns in the hierarchy that would apply to each scenario based on expert opinion. Such patterns subsets would be treated as the gold standard or "correct" set of patterns for evaluating novice performance. Furthermore, by interviewing experts, we sought to better understand how experts decide what security concepts are relevant to a particular system context. This information is fundamental to refining our pattern hierarchy.

The two banking scenarios were presented to each expert at the beginning of the interviews. We asked the participant to assume the role of a software analyst with the task of creating security requirements for the system. The actor playing the domain expert was introduced and the facilitator explained that he would be available for any questions or clarification that may be needed. The same domain expert was present during each interview to any questions the expert participant may have had.

Interview questions were planned out according to a script based on the ICM (described in Section VI). We used this model as a means to encourage the expert to ask questions that could later be used in the modification of our pattern hierarchy. For example, when expert participants began to consider an access control policy they would ask the domain expert questions such as, "What kinds of users are there?" or "When do you want users to be able to access the system?". We looked for these kinds of cues to construct a hierarchy using the method described in Section IV.

After the interview was completed, we asked the participant to go through the existing list of security requirements patterns in the hierarchy and affirm whether or not the pattern should be regarded for the scenario along with an explanation for inclusion or exclusion. Experts were also encouraged to add anything else they thought was relevant. Transcriptions of the interviews were analyzed along with any notes the experts provided in order to create a final Access Control hierarchy as seen in Figure 2. This resulted in the Authorization pattern [9] being placed at the root of the hierarchy above Access Control. We continue to refer to the hierarchy as an Access Control hierarchy due to our scenarios being focused on access control as well as consistency.

A subset of the patterns in the final Access Control hierarchy was also compiled for each scenario based on expert responses to be used in the novice interviews. To do this, we tallied the selections made by the experts resulting in Figures 3 and 4 . If at least five of the seven agreed on a pattern, we included it in the subset of correct patterns for the scenario. This resulted in 12 of 17 patterns for scenario 1 and 13 of 17 for Scenario 2. 


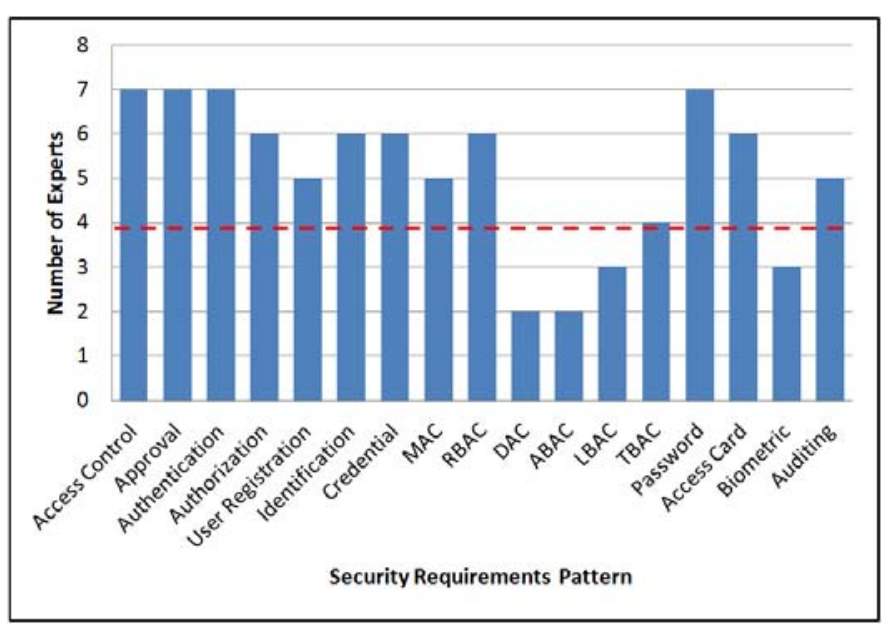

Figure 3. Scenario 1 expert pattern selection

\section{Novice Interviews}

Novice participants were interviewed individually and randomly placed into either a control or an intervention group. Members of both groups were asked to consider the same two scenarios given to the experts and were provided with the same domain expert present during the expert interviews. Participants for both groups were placed into the role of a software analyst and asked to select the most relevant patterns for each scenario from a list of the patterns from the Access Control hierarchy. Participants were informed that any information they provided would be made anonymous so as to encourage them to proceed naturally. The time to complete both scenarios was also recorded.

For the control group, only the list was given with no relations between the patterns as in the hierarchy. This group was provided with access to the Internet and asked to make the selections to the best of their ability. This gave us a baseline description of how well novices could do in pattern selection on their own.

The intervention group was presented with a questionnaire (described in Section VI) representing the hierarchy. A questionnaire was used in order to forgo any confusion involving training novices in the use of feature diagrams. The questionnaire was created by organizing the pattern hierarchy questions so that they could correspond to the same checklist of patterns given to the control group. Instructions placed after each question both directed the participants to appropriate consecutive questions and had the participant check off appropriate patterns for the scenario based on the hierarchy.

\section{E. Risks to Validity}

In order to make statistical comparisons between the two groups, we had to provide the control group with the same set of patterns to choose from as the intervention group. In the wild, it would be up to the user to select from all available patterns. By providing the control group with a list, we were forced to provide them with much of the work that would have been done by the hierarchy. We believe that the control group would have performed more poorly in both selection and

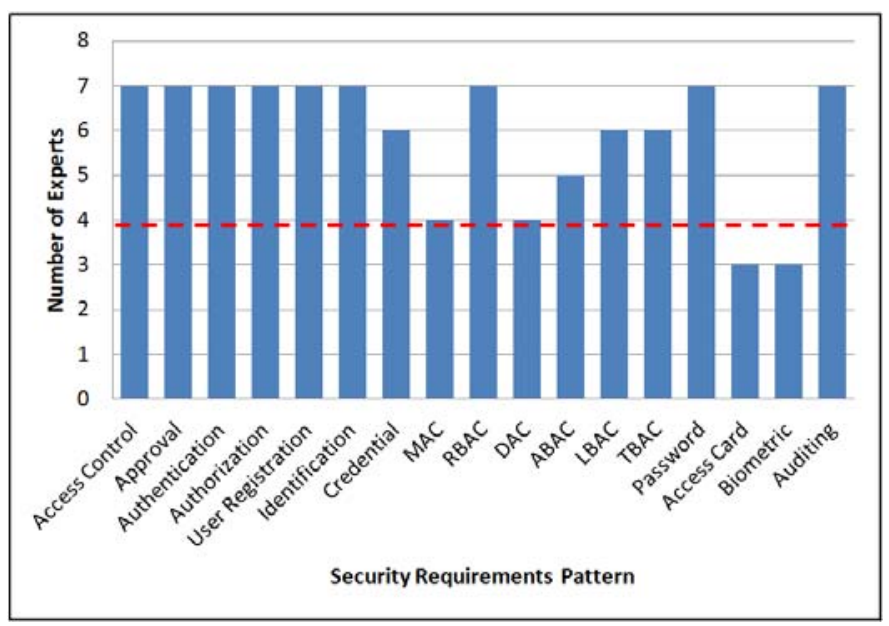

Figure 4. Scenario 2 expert pattern selection

speed without the provided list. This would actually further validate the use of pattern hierarchies.

We chose not to use the feature diagram representation of the hierarchy for our study due to the time constraints involved for training. Even with the feature diagram notation, the same questions must be answered as in the questionnaire. The feature diagram itself is useful for visualizing the flow between patterns; however the user must be familiar with the notation. We felt that the questions alone were enough for the user to gain the guidance provided by the hierarchy, and a properly-trained user would have only performed better. By providing the feature diagram representation to participants unfamiliar with it, we would have risked user error due to misunderstanding of the notation.

\section{INTERVIEW MATERIAL}

The following subsections include material used during novice and expert interviews. This includes scenarios, questionnaires, and scripts.

\section{A. Scenarios}

The following background was presented prior to any scenarios:

Military Credit Union, a new credit union, is partnering with Bank of USA and plans to open 30 branches in a new state. Members of Military Credit Union will have the ability to perform all normal banking transactions (deposit, withdrawal, transfer, check, etc.). Major loans will be issued by Bank of USA but can be applied for at either institution.

All new branches will need a total of 500 employees and serve about 2 million customers. Each branch has a branch manager, a financial advisor, a few account representatives, and about 10 tellers. All employees at the branch should be able to access client accounts. Tellers can only deal with simple deposits and withdrawals, all other transactions are to be completed by the account representatives. An internal instant messaging system will allow all employees to exchange messages with each other as well as with the employees at Bank of USA. The credit union's state headquarters will house an executive manager to oversee all branch operations, a loan officer to approve loan applications, 
and fund manager to review investments. Technicians will be available, in each city, to troubleshoot any technical problems but shouldn't be able to view client account information.

Scenario 1 was described as follows:

Customers will have access to a 24 hour ATM machine located directly outside of each branch location. In addition to these ATMs, Bank of USA has allowed Military Credit Union to use approximately 300 ATMs that are owned and operated by the bank so as to provide customers with more convenient access to their accounts. Military Credit Union customers will not incur any additional fees for using any Bank of USA ATM. Bank of USA will record the number of transactions completed on their ATMs and charge Military Credit Union accordingly at the end of each month.

Scenario 2 was described as follows:

Military Credit Union is transitioning their existing traditional banking system to a modern computerized one. This new system should handle all bank transactions and have the ability to transfer funds between accounts of both institutions. Online banking will also be available to customers who elect to enable these services. From any web enabled computer, customers will be able to log on to the system and manage their accounts. Balance transfers, automatic bill pay, and check management (cancel check, reorder check, etc.) are some of the features available through the online banking system. Customers will be able to change contact information (phone number, mailing address) as well as apply to open new accounts. An indoor kiosk at each branch will be provided to customers who wish to browse the credit union's web site. All major web browsers will be supported and special consideration will be taken when a customer accesses the site through a public computer (library) versus a private one (home office desktop).

\section{B. Expert Interview Script}

The following script was used in expert interviews to begin discussion. Questions were based on the ICM and, in many cases, clarification was required through the form of follow-on questions. Examples of such follow-on questions are included. (Who)

Who should have access to the system?

(Clarification and follow up may be
required)
Ex: Who are the users?
- $\quad$ Are there any other users that may
need access to the system?

(What-kinds-of)

What information should be shared between institutions?

(Clarification and follow up may be required)

Ex:

- Can you think of any other types of information?

(What - kinds-of)
What information should we protect to enforce information confidentiality and integrity?

(Clarification and follow up may be required)

(What-kinds-of)

What access information shall be kept to provide accountability?

(clarification and follow up may be required)

(How-to)

How do you authenticate?

(Clarification and follow up may be

required)

Ex:

[if password] $->$ (Follow-on/What -

kinds-of)

What kind of password shall we require?

[if biometric] $->$ (Follow-on/What -

kinds-of)

kinds of biometric scans should be used?

(fingerprint, face, iris, hand, etc.)

[if access card]-> (Follow-on/What kinds-of)

What kind of access card should be used? (active/passive, magnetic/radio)

(What-kinds-of)

What kind of threats do we want to

prevent?

(Clarification and follow up may be

required)

(When)

Are there any time constraints on access within this system?

(Clarification and follow up may be required)

(What-if)

If an employee gets moved to a different position what needs to be considered?

(Clarification and follow up may be required)

(How-to)

Describe the organization of an access control approach for this type of scenario?

(Clarification and follow up may be required)

(Follow-on)

Why would other approaches not be suitable for this scenario? 
(Clarification and follow up may be required)

(How-to)
Which access control policies and
enforcement techniques should be
considered for this scenario?
(Clarification and follow up may be
required)

(Follow-on)

Are there any benefits of choosing a

different technique?

(Clarification and follow up may be required)

-SCENARIO SPECIFIC QUESTIONS-

SCENARIO 1 QUESTIONS

(What-if)

What if someone loses his or her PIN?

(Clarification and follow up may be

required)

(What-if)

What if many invalid PINs have been attempted?

(Clarification and follow up may be

required)

(How-to)

What mechanisms should be employed to

ensure the security of the system?

(Clarification and follow up may be required)

(Follow-on)

How does this affect the usability and

availability of the system?

(Clarification and follow up may be

required)

\section{SCENARIO 2 QUESTIONS}

(What-if)

What if someone loses his or her username or password?

(Clarification and follow up may be

required)

(What-if)

What if many invalid username/password combinations have been attempted?

(Clarification and follow up may be required)
What mechanisms should be employed to ensure the security of the system? (Clarification and follow up may be required)

(Follow-on)

How does this affect the usability and availability of the system? (Clarification and follow up may be required)

\section{Novice Questionnaire}

The following questionnaire was used for the novice user portion of the study in lieu of a graphical hierarchy:

1) Authorization

a) Are there parts of the system where permission to gain access to a resource cannot be automated (i.e., an actual person must be consulted for the authorization)?

If yes, check "Approval".

b) Will access to the system or resources need to be restrictive on a selective basis (example: only members have access to member data)?

If yes, check "Access Control"

c) Check "Authorization" and "Auditing".

2) Access Control

If "Access Control" is not checked, proceed to question 3.

a) Check "Authentication".

b) Can users of the system be categorized into roles that will have different access privileges? A role can be described as job function or title which defines an authority level. (examples of roles: student, professor, department chair, administrator, guest etc)?

If yes, check "Role Based Access Control".

c) Must user, session, or resource attributes be used to restrict or grant access to resources? An attribute can be described as a quality, trait, or characteristic. (examples: time, location, user age, desired action)

If yes, check "Attribute Based Access Control".

d) Choose at most one (either none or 1) of the following (2.d.i or 2.d.ii)

i) Can resources be assigned labels by the system so that users are given clearance to access resources based on levels of clearance (i.e., resource owners cannot change the clearance needed to access the resource)?

If yes, check "Mandatory Access Control".

ii) Can access to resources be regulated by the owner of the resource?

If yes, check "Discretionary Access Control".

3) Authentication

If "Authentication" is not checked, proceed to question 4.

a) Check the following three: "Identification", "User Registration", and "Credential".

(How-to) 


\begin{tabular}{|c|c|c|c|c|c|c|c|c|c|}
\hline & \multicolumn{2}{|c|}{ Average Success Rate (\%) } & \multirow{2}{*}{$\begin{array}{l}\text { Increase } \\
\left(\bar{x}_{I}-\bar{x}_{C}\right) \\
\end{array}$} & \multicolumn{2}{|c|}{ Standard Deviation (s) } & \multicolumn{2}{|c|}{ Sample Size (n) } & \multirow{2}{*}{$\begin{array}{c}\text { Significance } \\
\text { (p-value) }\end{array}$} & \multirow{2}{*}{$\begin{array}{l}\text { Effect Size } \\
\text { (Cohen's d) }\end{array}$} \\
\hline & Control $\left(\overline{\mathrm{x}}_{\mathrm{C}}\right)$ & Intervention $\left(\overline{\mathrm{X}}_{\mathrm{I}}\right)$ & & Control & Intervention & Control & Intervention & & \\
\hline Scenario 1 & 60.78 & 82.92 & 22.13 & 14.70 & 12.41 & 17 & 16 & 0.00003 & 1.63 \\
\hline Scenario 2 & 72.08 & 82.75 & 10.66 & 11.21 & 7.09 & 16 & 17 & 0.002 & 1.16 \\
\hline
\end{tabular}

4) Credential

If "Credential" is not checked, proceed to question 5.

a) Choose any two of the following (4.a.i through 4.a.iv)

i) Do the majority of users NOT have sufficient knowledge to correctly implement and use certificate-based or public key authentication techniques?

If yes, check "Password".

ii) Are the following two statements true: (1) Is usability (e.g., speed, ease of remembering) a large factor? (2) Will access be limited to locations where specific hardware can be installed?

If yes, check "Access Card".

iii) Are the following two statements true: (1) Does security outweigh the cost of implementing an authentication mechanism? (2) Will access be limited to locations where specific hardware can be installed?

If yes, check "Biometric".

iv) If only one of the above three can be chosen, check "Security Question".

5) Attribute Based Access Control

If "Attribute Based Access Control" is not checked, this concludes the questionnaire.

a) Should access to resources be restricted based on time of access (e.g., time between accessing, time of access, total time accessed)?

If yes, check "Time Based Access Control".

b) Should access to resources be restricted based on location accessed from?

If yes, Check "Location Based Access Control".

\section{RESULTS AND OBSERVATIONS}

Here, we discuss the knowledge we were able to extract from the experts as well as how our hierarchy affected novice decisions.

\section{A. Expert Knowledge Goes Beyond Patterns}

An important contribution of patterns is the reuse of expert knowledge. Our research asks if expert knowledge could go beyond individual patterns and expand to pattern organization and selection through the use of pattern hierarchies. Based on our literature review, interaction with experts, and what we were able to produce with that knowledge, we found that expert knowledge can be applied in a larger scale through our hierarchies.

We used 14 patterns from textbooks and research papers prior to our interaction with experts [9, 12]. From the seven interviews we were able to expand that number to 17 from the additions the experts included to our checklist of patterns. The additions (auditing, time-based access control (TBAC), location-based access control (LBAC), and security question) were not in the form of explicit patterns, but fit into the hierarchy as supplemental security topics related to the existing patterns. We assert that this implies the potential for yet-to-be-created patterns.

The links between patterns which formed the pattern hierarchy were produced by integrating information already present in patterns (e.g., "Related Patterns" sections) and information gained from expert interviews. Generally, as experts described what kind of security requirements were important for the scenarios they would ask the domain expert questions for clarification. For example, when discussing attribute-based access control (ABAC) [39], the ICM urged us to pose the follow-on question: "when should users have access to the system?” Experts would often respond by asking the domain expert if there would be times when employees would not be at work and if they should have access during those times. Depending on the answers to these questions, a need for a corresponding requirements pattern would be necessary. For this example, the domain expert indicated that there are parts of the day when no employees are at work and thus should not be able to access the system. This triggered the expert to begin considering requirements that would be part of a TBAC pattern. The resulting relation between ABAC and TBAC could now be represented with the question: "are there times when users should not have access to the system?” This is represented in Figure 2 by the optional connection between these two patterns. The question itself would become part of the solution space of the ABAC pattern.

The experts would often give stipulations for some security requirements. For example, biometrics should only be used with consent of the user. These would be handled similarly. Whether or not the stipulation was viable would be posed in the form of a question and applied to the hierarchy. Furthermore, the question of viability could have implications on the relevant quality attributes to be represented in the Forces portion of the resulting pattern. For this example, usability would be a contributing factor.

\section{B. Pattern Hierarchies are Efficient and Usable}

Novice results were measured by correct pattern choice and time to completion. In both cases, the intervention group performed better than the control group.

Regarding correct pattern choice, novices were graded depending on if they made the same decision to include or exclude a particular pattern as defined by the gold standard pattern sets derived from expert decisions described in Section V-C. For example, Scenario 1 DAC was not included by experts, so if a novice user chose to include the pattern, then it was marked as incorrect. Points were not deducted for incorrect answers. A majority of the experts expressed the need for two-factor authentication [40] for both scenarios. To accommodate this, a correct choice of authentication patterns (password, biometric, access card, and security question) 
consisted of any two. If a participant chose only one of those patterns, they were not awarded points. This selection scheme is apparent in Figure 2 with the range of necessary patterns denoted within the exclusive-or arc with "[2]".

Before computing the results of our study, we removed outliers by using Iglewicz and Hoaglin's modified Z-score with median absolute deviation method (MAD) [41]. The $M A D$ for each group was calculated with:

$$
M A D=\operatorname{median}\left(\left|x_{i}-\tilde{x}\right|\right)
$$

Modified Z-scores $\left(M_{i}\right)$ for each score were calculated with:

$$
M_{i}=\frac{0.6755 *\left(x_{i}-\tilde{x}\right)}{M A D}
$$

Where $x_{i}$ represents individual scores and $\tilde{x}$ is the median. 0.6755 is the multiplier used for outlier detection recommended by Iglewicz and Hoaglin for samples of our size. For this method, values of $M_{i}$ greater than $(3.5 * M A D)$ were removed as statistical outliers. This resulted in the removal of two participants.

Table 1 describes the quantitative results of the study regarding successful pattern choice by novice users. For both Scenario 1 and Scenario 2 novices were more successful at making the same choices as experts when using the pattern hierarchy with a success rate increase over the control group of $22.13 \%$ and $10.66 \%$ respectively. Regarding the lower increase for Scenario 2, we found that fewer users asked the domain expert questions for that scenario. We took this as an implication of misappropriated familiarity with the situation. Unless a user has sufficient experience with the situation, it is necessary for them to ask questions in order to properly specify requirements.

We used a standard t-test to measure the significance of our results. We tested the null hypothesis $\left(H_{0}\right)$ that the mean population success rates for users without the hierarchy $\left(\mu_{C}\right)$ and users with the hierarchy $\left(\mu_{I}\right)$ were equal:

$$
H_{0}: \mu_{C}-\mu_{I}=0
$$

The p-values produced by the t-tests indicate that the null hypothesis could be rejected for both scenarios and that the results of our study were statistically significant.

As an indicator of the strength of our sample size, we calculated effect size using Cohen's d [42]. This was done by taking the difference between the mean sample success rate for the control group $\left(\bar{x}_{C}\right)$ and the mean sample success rate for the intervention group $\left(\bar{x}_{I}\right)$ divided by the average standard deviation for both groups, $s_{I C}$ :

$$
d=\frac{\bar{x}_{I}-\bar{x}_{C}}{s_{I C}}
$$

Based on Cohen's conventions, the values for both Scenario 1 and Scenario 2 indicated a large effect size. This implied meaningful results regardless of our sample size.
Efficiency in terms of completion time was recorded for both groups. Table 2 shows an average decrease in selection time of more than $80 \%$ for the group using the pattern hierarchy. We attribute this increase in selection speed to the guidance the hierarchy provided. Without the hierarchy, the control group was forced to use their previous knowledge and the Internet to make decisions. Participants were able to make decisions faster while covering the same amount of patterns with the aid of the straightforward questions provided by the hierarchy. We calculated statistical significance and effect size with the same methods used for success rates. Both values implied statistical significance and meaningful results.

\section{RELATED WORK}

Researchers have been documenting security patterns for decades, and there have been similar efforts to increase the usability of patterns [16]. We now review and discuss the similarities of these related efforts in the security pattern domain, and how they differ from our own work.

Romanosky extends the work done by Yoder and Barcalow [43] by introducing an additional eight security patterns [44]. Romanosky adopts the standardized pattern template originally developed by the GoF, which includes: Problem, Forces, Solution, and Consequences. Their template also uses the following additional elements not included in our approach: Alias, Motivation, Example, and Related Patterns. Although many of the element titles are identical in both templates, we assign slightly different meanings. For example, Romanosky's Solution section is a textual description of a step or series of steps that, when applied, can mitigate the problem. In contrast, our Solution is represented as a series of questions derived using the ICM, to guide users to other patterns and to generate requirements based on answers to these questions as described in Section III. Additionally, questions in Romanosky's approach do not direct readers to related patterns nor do they provide a customized solution based on their answers. Moreover, our Forces section is used to influence the types of questions contained within the Solution, by surfacing trade-offs that arise from other quality attributes.

Based on the analysis of 220 security patterns, Heyman et al. investigate possible improvements that can be made to increase their usability [45]. Their major conclusions include that the quality of patterns would greatly benefit by the adoption of a common documentation template, and that the construction of a "pattern inventory" would make pattern selection a less daunting task. As opposed to having a general inventory, our approach aims to contextualize each pattern in relation to other relevant patterns through the ICM and Forces. In this way, the pattern user discovers these other patterns as they become relevant and while the pattern user iterates to build a solution from each pattern. 


\begin{tabular}{|c|c|c|c|c|c|c|c|c|c|}
\hline \multicolumn{2}{|c|}{ Average Completion Time (minutes) } & \multirow{2}{*}{$\begin{array}{l}\text { Decrease } \\
\left(\overline{\mathrm{X}}_{\mathrm{C}}-\overline{\mathrm{X}}_{\mathrm{I}}\right)\end{array}$} & \multirow{2}{*}{ Speedup } & \multicolumn{2}{|c|}{ Standard Deviation (s) } & \multicolumn{2}{|c|}{ Sample Size (n) } & \multirow{2}{*}{$\begin{array}{c}\text { Significance } \\
\text { (p-value) }\end{array}$} & \multirow{2}{*}{$\begin{array}{l}\text { Effect Size } \\
\text { (Cohen's d) }\end{array}$} \\
\hline Control $\left(\overline{\mathrm{X}}_{\mathrm{C}}\right)$ & Intervention $\left(\overline{\mathrm{X}}_{\mathrm{I}}\right)$ & & & Control & Intervention & Control & Intervention & & \\
\hline 28.56 & 15.82 & 12.74 & 1.81 & 11.85 & 4.95 & 16 & 17 & 0.00037 & 1.52 \\
\hline
\end{tabular}

Table 2. Efficiency

Kienzle and Elder address some of the same problems we address with the security patterns landscape that Heyman et al. documented [46]. Specifically, they address the creation of a common pattern template and pattern repository. Like our template, their common pattern template was derived from the GoF template. Kienzle and Elder augmented the GoF template by including additional security-specific elements. These additions make the patterns somewhat lengthy and, as discussed in the previous paragraph, our Solution section provides more than just a textual description of a procedure. Using their template, they have documented a total of 26 security patterns in a patterns repository.

Hafiz et al. introduce an approach for organizing and describing the relationship between patterns using directed acyclic graphs [16]. Their approach uses a hierarchical scheme based on threat models in order to classify security patterns. Patterns which target similar problems are grouped together similarly to our hierarchical approach. With these grouped patterns, the researchers analyzed the order in which they would be applied to describe their relationships and position in the hierarchy. Dotted line arrows and solid line arrows show relationships between patterns for preventing 'tampering and DoS (denial of service)' and 'Escalation of Privilege', respectively. The authors admit that their pattern language is still a work in progress, is hard to read, and can be "large and intimidating." Based on the results from our empirical study, we believe that the use of feature diagrams provides a more usable method of pattern selection for novice users.

\section{FUTURE WORK}

A natural next step would be to complete the Access Control hierarchy used in our user study. We mentioned in Section IV that there were indications of yet unimplemented patterns that would fit our hierarchy. The Auditing and TBAC patterns are examples where experts explored such areas of access control but there were no existing patterns, at least that we found, that fit the description. We hope to fill these spots with either existing or newly-mined patterns. Based on comments by experts in our user study, we believe there is also room for expansion of the hierarchy to include patterns for encryption and sessions [12]. We will use the same approach that we used to create the hierarchy to add appropriate patterns as they are discovered for these areas.

We created a digital repository of the security patterns collected for our research. Patterns exist as individual entries with no connections to other patterns in the repository's current state. We plan to modify this repository to have the ability to relate security requirements patterns through the use of pattern hierarchies. If we can provide public access to such a hierarchy-based repository we would not only provide the benefit of pattern hierarchies to software engineers, but also receive valuable feedback to further our research and expand the hierarchies themselves.

Up to this point, our research in pattern hierarchies has worked to show that such a method exists to store and use patterns. In producing and empirically testing the Access Control hierarchy, we have shown that such a structure can be created and is usable. Next, we will work to formalize a method for hierarchy creation. In doing so, we will allow for the creation of more hierarchies in different domains or in different stages of software development.

\section{CONLUSION}

Using security requirements patterns can greatly reduce the time spent in the requirements elicitation phase of software design only if the pattern user can be sure he or she has identified the correct pattern to apply. Our comparison of pattern selection with and without the hierarchy showed statistically significant and meaningful results. This upholds our hypothesis that pattern hierarchies can be created to allow engineers to make more expert-like decisions in efficient time. Using this method, software engineers can be more confident in the completeness of their requirements specifications. We feel that, based on our results, incorporation of pattern hierarchies with existing patterns can better facilitate knowledge transfer in the requirements engineering domain.

\section{ACKNOWLEDGMENT}

We thank Hanan Hibshi, Laurie Williams, and Maria Riaz for their insightful comments. We thank Daniel Amyot, Xavier Franch and other RePa'12 participants for their contributions in the initial brainstorming on requirements patterns. This research was funded by Army Research Office (Award \#W911NF-09-1-0273).

\section{REFERENCES}

[1] S. Konrad and B. H. Cheng, "Requirements patterns for embedded systems," RE'02, p. 127, 2002.

[2] C. Alexander, S. Ishikawa, M. Silverstein, M. Jacobson, I. FiksdahlKing, and S. Angel, A pattern language, Oxford University Press, 1977.

[3] M. Lin and H. Guo, "Present situation and development of single signon tehnology,” Journal of Computer Applications, pp. 248-250, 2001.

[4] R. Sandhu, E. Coyne, H. Feinstein, and C. Youman. Role-Based Access Control Models. IEEE Compute 29(2), pp. 38-47, 1996.

[5] K. Beck, R. Crocker, G. Meszaros, J. Vlissides, J. O. Coplien, and L. Dominick, "Industrial experience with design patterns," ICSE'96, pp. 103-114, 1996

[6] G. McGraw, "Testing for security during development: why we should scrap penetrate-and-patch," IEEE T. Aero. Elec. Sys. 13(4), 1998.

[7] B. Boehm, "Software engineering economics," TSE10, pp. 4-21, 1984.

[8] N. Yoshioka, H. Washizaki, and K. Maruyama, "A survey on security patterns,” Progress in Informatics, No.5, pp. 35-47, 2008.

[9] S. Withall, Software Requirements patterns, Microsoft Press, 2007.

[10] D. Kienzle, M. C. Elder, D. Tyree, and J. Edwards-Hewitt, "Security patterns repository version 1.0,” DARPA, Washington DC, 2002. 
[11] M. A. Jalil and S. A. M. Noah, "The difficulties of using design patterns among novices: an exploratory study," ICCSA'07, 2007.

[12] M. Schumacher, E. Fernandez-Buglioni, D. Hybertson, F. Buschmann, and P. Sommerlad, Security patterns: integrating security and systems engineering, John Wiley \& Sons, 2006.

[13] M. Weiss and H. Mouratidis, "Selecting security patterns that fulfill security requirements," $16^{\text {th }}$ ICSE'08, pp. 169-172, 2008.

[14] E. Gamma, R. Helm, R. Johnnson, and J. Vlissides, Design patterns: elements of reusable object-oriented software, Addison-Wesley, 1994.

[15] T. D. Breaux, H. Hibshi, A. Rao, and J-M. Lehker. “Towards a framework for pattern experimentation: understanding empirical validity in requirements engineering patterns.” RePa'12, pp. 41-47, 2012.

[16] M. Hafiz , P. Adamczyk , R. E. Johnson, "Growing a pattern language (for security),” Onward!'12 pp.139-158, 2012.

[17] K. Kang, S. Cohen, J. Hess, W. Novak, and A. Peterson, "Featureoriented domain analysis (FODA) feasibility study,” Carnegie Mellon Univ., CMU/SEI-90-TR-021, 1990.

[18] P. Zave. (2004). FAQ sheet on feature interactions [Online]. Available: www.research.att.com/ pamela/faq.html

[19] D. Batory, "Feature models, grammars, and propositional formulas," $9^{\text {th }}$ international conference on Software Product Lines, pp. 7-20, 2005.

[20] D. Bell and L. LaPadula. Computer Security Model: Unified Exposition and Multics Interpretation, MITRE Corp., ESD-TR-75-306, 1975.

[21] G. Graham and P. Denning. "Protection-principles and practice,” AFIPS Spring Joint Computer Conference, pp. 417-429, 1972.

[22] P-Y. Schobbens, P. Heymans, J-C. Trigaux, "Feature diagrams: a survey and a formal semantics,”RE'06, pp.139-148, 2006.

[23] C. Potts, K. Takahashi, and A. I. Antón, "Inquiry-based requirements analysis,” IEEE Software, pp. 21-32, 1994.

[24] J. Mylopoulos and J. Castro, "Tropos: a framework for requirementsdriven software development," Information Systems Engineering: State of the Art and Research Themes, pp. 261-273, 2000.

[25] M. Jackson, Problem frames; analyzing and structuring software develoment problems, Addison-Wesley, 2001.

[26] C. Palomares, C. Quer, X. Franch, C. Guerlain, and S. Renault, "A catalogue of non-technical requirement patterns," $\mathrm{RePa} 12$, pp. 1-6, 2012.

[27] D. Dietrich and J. M. Atlee, “A pattern for structuring the behavioral requirements of features of an embedded system,” $R e P a ' 12$, pp. 1-7, 2012.

[28] K. Yskout, R. Scandariato, and W. Joosen, "Does organizing security patterns focus architectural choices?,” ICSE’12, pp. 617-627, 2012.

[29] R. Slavin, H. Shen, and J. Niu, "Characterization and Boundaries of Security Requirements patterns,” $\mathrm{RePa} 12, p p$. 48-53, 2012.

[30] J. Anderson, "Information security in a multi-user computer environment," in Advances in Computers (12), pp. 1-35, 1973.

[31] S. Lauesen, Software Requirements: Styles and Techniques, Pearson Education Limited, 2002.

[32] V. Krishnan, M. V. Tripunitara, K. Chik, and T. Bergstrom, "Relating declarative semantics and usability in access control,” SOUPS'12, pp. 14, 2012.

[33] R. Crook, D. Ince, and B. Nuseibeh, “On modelling access policies: relating roles to their organisational context,” RE'05, pp. 157-166, 2005.

[34] M. Koch, F. Parisi-Presicce, "Formal access control analysis in the software development process,” FMSE’03, pp. 67-76, 2003.

[35] H. Hibshi, R. Slavin, J. Niu, and T.D. Breaux, "Rethinking security requirements in RE research,” Tech. Rep. Report CS-TR-2014-001, Univ. Texas at San Antonio, 2014.

[36] J-M. Lehker. (2014). Security Pattern Repository [Online]. Available: http://sefm.cs.utsa.edu/repository/patterns/

[37] S. S. Council. Payment Card Industry (PCI) Data Security Standard, 2nd ed., 2010.

[38] D. Kim, P Mehta, and P Gokhale. "Describing access control models as design patterns using roles,"PLoP'06. 2006.

[39] L. Wang, D. Wijesekera, S. Jajodia, “A logic-based framework for attribute based access control,” FMSE'04, pp. 45-55, 2004.
[40] M.L. Das, "Two-factor user authentication in wireless sensor networks," TWC'09, pp. 1086-1090, 2009.

[41] B. Iglewicz and D. C. Hoaglin (1993), "How to detect and handle outliers", in The ASQC Basic References in Quality Control: Statistical Techniques, vol. 16, ASQC Quality Press, 1993.

[42] J. Cohen, “A power primer,” Psychological Bulletin, 1992, pp. 155-159, 1992.

[43] J. Yoder and J. Barcalow, "Architectural patterns for enabling application security,” PLoP'97, pp. 1-37, 1997.

[44] S. Romanosky. Security design patterns part 1. http://citeseer.ist.psu.edu/575199.html, Nov 2001.

[45] T. Heyman, K. Yskout, R. Scandariato, and W. Joosen, "An analysis of the security patterns landscape," SESS '07. p. 3, 2007.

[46] D. M. Kienzle and M. C. Elder, "Security patterns for web application development, final technical report”, 2003. 